\title{
Extending modified module analysis to include correct responses: Analysis of the Force Concept Inventory
}

\author{
Jie Yang, ${ }^{1}$ James Wells $\odot,{ }^{2}$ Rachel Henderson, ${ }^{3}$ Elaine Christman, ${ }^{1}$ \\ Gay Stewart, ${ }^{1}$ and John Stewart $\oplus^{1, *}$ \\ ${ }^{1}$ West Virginia University, Department of Physics and Astronomy, Morgantown, \\ West Virginia 26506, USA \\ ${ }^{2}$ College of the Sequoias, Science Division, Visalia, California 93277, USA \\ ${ }^{3}$ Michigan State University, Department of Physics and Astronomy, East Lansing, Michigan 48824, USA
}

(Received 24 January 2020; accepted 13 April 2020; published 27 April 2020)

\begin{abstract}
Brewe, Bruun, and Bearden first applied network analysis to understand patterns of incorrect conceptual physics reasoning in multiple-choice instruments introducing the module analysis for multiple-choice responses (MAMCR) algorithm. Wells et al. proposed an extension to the algorithm which allowed the analysis of large datasets called modified module analysis (MMA). This method analyzed the network structure of the correlation matrix of the responses to a multiple-choice instrument. Both MAMCR and MMA could only be applied to networks of incorrect responses. In this study, an extension of MMA is explored which allows the analysis of networks involving both correct and incorrect responses. The extension analyzes the network structure of the partial correlation matrix instead of the correlation matrix. The new algorithm, called MMA-P, was applied to the FCI and recovered much of the structure identified by MMA. The algorithm also identified sets of correct answers requiring similar physical reasoning reported in previous studies. Beyond groups of all correct and all incorrect responses, some groups of responses which mixed correct and incorrect responses were also identified. Some of these mixed response groups were produced when a correct response was selected for incorrect reasons; some of the groups were related to the gender unfair items previously reported for the FCI.
\end{abstract}

DOI: 10.1103/PhysRevPhysEducRes.16.010124

\section{INTRODUCTION}

The structure of students' conceptual understanding of physics and the evolution of that understanding has been an important research strand within physics education research (PER) since its inception. Research into student understanding has often relied on multiple-choice conceptual instruments such as the Force Concept Inventory (FCI) [1] and the Force and Motion Conceptual Evaluation (FMCE) [2]. Quantitative research examining these instruments began shortly after their introduction [3]. Recently, a new class of quantitative methods using network analysis has been applied to further understand common student difficulties with mechanics [4,5].

Network analysis is a broad and powerful set of analytic techniques applicable to situations as diverse as identifying drought resistant genes in plants [6] and exploring the status hierarchies of teenagers [7]. A network is formed of

\footnotetext{
*jcstewart1@mail.wvu.edu
}

Published by the American Physical Society under the terms of the Creative Commons Attribution 4.0 International license. Further distribution of this work must maintain attribution to the author(s) and the published article's title, journal citation, and DOI. nodes and edges where edges connect pairs of nodes. Edges can have numerical weights that represent properties that influence the strength of the relationship. Network analysis seeks to characterize the structure of the network. One important type of structure is the identification of groups of nodes that are more connected to each other than they are to other nodes in the network. These subgroups are called "communities" or "modules" interchangeably; we will call them communities to conform with the naming conventions of the "igraph" package [8] in the "R" software system [9] which was used for this analysis.

A substantial body of research has investigated differences in the conceptual performance of groups underrepresented in physics $[10,11]$; the majority of this work has examined differences between men and women [12]. The new network analytic techniques have been used to explore differences in the structure of incorrect reasoning of men and women [5]. We continue this practice and report results disaggregating men and women.

\section{A. Research questions}

The network analytic method used in this work extends the modified module analysis (MMA) introduced by Wells et al. [5] as an extension to the original algorithm pioneered 
by Brewe, Bruun, and Bearden [4]. Both methods were only productive when applied to the incorrect answers in the FCI; the correct answers formed a single tightly connected community that prevented identification of additional structure. Modified module analysis is described in detail in Sec. IC. The purpose of this study is to explore an extension of MMA to include correct responses; this extension is called MMA-P. This work was performed using the same dataset as the previous MMA study; this dataset was also used by Traxler et al. to demonstrate that a subset of the items in the FCI is substantially unfair to women [13]. This study will use the results of the MMA-P algorithm to further explore this unfairness.

This study explored the following research questions:

RQ1 How can modified module analysis be extended to include correct responses?

RQ1 Are the communities detected by the extended algorithm productive in furthering the understanding of the FCI? How are the communities identified by the extended algorithm related to structures identified in previous studies?

RQ3 How do the incorrect communities change between the pretest and the post-test?

RQ4 How do the communities detected differ between men and women?

\section{B. Prior studies}

This work draws heavily on two prior studies of the FCI performed on the same dataset as used in this study. These studies will be referenced as Study 1 and Study 2 in this work.

\section{Study 1: Modified module analysis}

Brewe, Bruun, and Bearden [4] first introduced network analysis to PER as module analysis for multiple-choice responses (MAMCR). Their initial work was applied to a sample containing only 143 FCI student responses. Wells et al. [5] sought to replicate this study with a large sample of FCI pretest and post-test responses $\left(N_{\text {pre }}=4509\right.$ and $\left.N_{\text {post }}=4716\right)$ and found the original MAMCR method did not scale to large datasets. To extend network analysis to large datasets, they introduced a modification of the original MAMCR algorithm called modified module analysis. The Wells et al. [5] study will be referenced as Study 1 in this work.

In MMA, the network is formed of nodes representing responses to the FCI. For example, the network contains a node representing the selection of response D to FCI item 2, node 2D. The edges in the network connect nodes that are correlated above some threshold; in Study 1, $r>0.2$ was used as the threshold where $r$ is the correlation coefficient. This threshold was selected because it generated a generally disconnected network where the sub-networks showed meaningful structure. The correlation coefficient is used as the weight of the edge. For example, if responses
2D and 5E have a correlation coefficient of $r=0.25$, then the network will contain an edge between nodes $2 \mathrm{D}$ and $5 \mathrm{E}$ with weight 0.25 . For productive analysis using MMA, nodes representing correct responses must be removed. If these nodes were included, they formed a densely connected community which obscured other structures.

Study 1 identified 9 groups of incorrect answers on the pretest and 11 groups on the post-test. In most cases, these groups could be explained as common misconceptions using the taxonomy of misconceptions introduced with the publication of the FCI [1] and refined by Hestenes and Jackson [14]. A subset of the incorrect communities was the result of the use of item blocks in the FCI. An item block is a group of items that refers to the same physical system or are written with a common stem. Most of the pretest and post-test groups identified were the same for men and women. The groups identified also had little relation to the gender unfair items identified by Traxler et al. [13]. Study 1 concluded that the differences in the structure of incorrect physical reasoning between men and women did not explain the gender differences reported for the FCI or the unfairness of the items in the instrument.

Study 1 was recently replicated by Wells et al. for the FMCE [15]. Incorrect responses representing the same misconception were identified in the same community demonstrating the algorithm is productive for examining instruments beyond the FCI. The incorrect communities identified, however, were often different for men and women both pre- and postinstruction as was the change in the communities from the pretest to the post-test.

\section{Study 2: Multidimensional item response theory and FCI}

In Study 2, Stewart et al. [16] used constrained multidimensional item response theory (MIRT) to develop a detailed model of the physical reasoning needed to correctly solve the FCI. Study 2 identified a number of subgroups of items in the FCI requiring similar physical reasoning for their solution. These subgroups were used extensively in Study 1 because the incorrect communities found by MMA were often well aligned with the groups of items requiring similar correct reasoning identified in Study 2. These subgroups were $\{4,15,16,28\},\{5,18\},\{6,7\}$, and $\{17,25\}$. Study 2 showed that the practice of blocking items was generating correlations between items not related to the physical principles tested and, as such, retained only the first item in an item block. The item blocks in the FCI are $\{5,6\},\{8,9,10,11\},\{15,16\}$, and $\{21,22,23,24\}$. While items $\{25,26,27\}$ are not explicitly blocked, both items 26 and 27 refer to item 25 and, therefore, these three items should be treated as an item block.

Wells et al. application of MMA to the FMCE [15] also relied on a constrained MIRT analysis of the instrument to identify items requiring similar physical reasoning [17]. 
As in Study 1, incorrect answer communities were often formed of items requiring the same correct reasoning.

\section{E. Results of prior research}

Prior research into the structure of the FCI was thoroughly summarized in Study 2, research into the misconceptions measured by the FCI in Study 1, and issues of the gender fairness and how they relate to the FCI in Traxler et al. [13]. These fairly extensive research strands are summarized below; readers interested in more detail are directed to these previous works.

\section{Exploratory analyses of the FCI}

Hestenes, Wells, and Swackhammer decomposed the concept of force into six conceptual dimensions and provided descriptions of the concepts each FCI item was intended to measure [1]. However, attempts to extract this factor structure using exploratory factor analysis (EFA), which uses correlations between all items to select groups that measure the same idea, proved unsuccessful. Huffman and Heller identified only two of these six factors, Newton's 3rd law and "Kinds of Forces," in their principal component analysis of a sample consisting of 145 high school students. The only factor identified in a sample of 750 university students was kinds of forces [3].

Scott, Schumayer, and Gray found that while only one factor explained much of the variance in an EFA study of the FCI post-tests of 2150 college students, 5 factors were needed for an optimal model [18]. Scott and Schumayer identified a similar, but not identical, five-factor model when using MIRT on a related dataset, confirming their model and suggesting that traditional EFA and MIRT are complementary techniques [19]. Semak et al. applied factor analysis to the FCI pretest and post-test responses of 427 college students and found that an optimal model included five factors for the pretest and six for the post-test [20]. Study 2 conducted an exploratory MIRT analysis of college post-test data and reported that a nine-factor model was optimal [16].

\section{Gender and the FCI}

Differences between the performance of men and women on the FCI and FMCE have been broadly reported with men outperforming women by $13 \%$ on pretests and $12 \%$ on post-tests. Many explanations have been advanced to explain these differences including differences in high school physics class election [21-23], cognitive differences [24-27], and psychocultural factors including mathematics anxiety [28,29], science anxiety [30-32], and stereotype threat [33].

All the above explanations locate the source of the performance differences with the students, a separate body of research has shown multiple items in the FCI are unfair to women, and some to men [34-36]. An item is unfair if men and women with the same general ability with the material score differently on the item. Gender differences in some FCI items have been reported for many years. Recently Traxler et al. [13] provided convincing evidence that 5 FCI items, items 14, 21, 22, 23, and 27, were substantially unfair to women using samples from three institutions. In their largest sample, differential item function (DIF) theory [37] identified a total of eight items with large DIF; six were unfair to women $(12,14,21,22,23,27)$ and two to men (items 9 and 15). The problematic items had generally, but not consistently, been identified in earlier work [34-36].

\section{The structure of knowledge}

MAMCR, MMA, and MMA-P all attempt to identify structure within student responses to a multiple-choice instrument. MAMCR and MMA only investigate incorrect responses and, thus, attempt to find coherent patterns of incorrect reasoning. Understanding common difficulties shared by many students in learning Newtonian mechanics has been an important area of research in PER since its inception [38-44]. This research led to a systematic exploration of students' understanding of Newton's laws and its epistemological development [2,45-48].

From these investigations and research outside of PER, theoretical frameworks of the structure of students' conceptual knowledge of physics emerged. Within PER, patterns of incorrect answering have often been characterized as "misconceptions," consistently applied incorrect reasoning specific to the physical concept tested. Other theories have been advanced to explain incorrect reasoning; two of the most prominent are the knowledge-inpieces framework $[49,50]$ and the ontological categories framework [51-53].

The knowledge-in-pieces framework models student knowledge as composed of small pieces of reasoning that are applied either independently or collectively to solve a problem. Models involving small fragments of reasoning have been advanced by many authors and have been called phenomenological primitives ( $p$ prims) [49,50], resources [54-56], and facets of knowledge [57]. In the knowledgein-pieces framework, what PER has called misconceptions are the result of problems activating the same collections of inappropriately applied knowledge pieces. In this framework, misconceptions are not "resolved," rather students" frameworks must be refined to apply to the situation in the problems. The ontological categories model suggests that incorrect student answers are a result of misclassification of a physical concept. For example, force may be misclassified as a substance.

Scherr [58] provides a concise definition of both the misconception view and the knowledge-in-pieces view that we will adopt for this work. The misconception view is "a model of student thinking in which student ideas are imagined to be determinant, coherent, context-independent, 
stable, and rigid" [58]. The knowledge-in-pieces framework models student conceptual ideas "as being at least potentially truth-indeterminate, independent of one another, context-dependent, fluctuating, and pliable" [58].

The research in the current work most closely aligns with the misconception and knowledge-in-pieces views. Study 1 and Wells et al. [15] both observed that communities of incorrect answers are generally formed of items requiring the same physical reasoning for their solution and shared a common misconception from the taxonomy of Hestenes and Jackson [14]. Because the incorrect reasoning is applied across multiple similar problems, the misconception view may be more appropriate for the incorrect answer communities identified by MMA or MMA-P. The FCI was developed partially to probe common misconceptions [1], so the finding that this framework is more appropriate is not a surprise. Network analysis could potentially identify $p$ prims as consistently applied reasoning divorced from the physical context, but thus far this type of community has not been observed.

\section{METHODS}

\section{A. Instrument}

The FCI is a 30-item instrument designed to assess a student's conceptual understanding of topics in Newtonian mechanics including one- and two-dimensional kinematics and Newton's laws. The FCI's coverage of Newtonian mechanics is not exhaustive; the instrument contains no questions on common topics from introductory mechanics courses such as conservation of momentum and energy [1]. Each item includes four incorrect responses with distractors intended to elicit common student misconceptions as well as one correct response. This study uses the revised FCI released in 1995 [59], which is available at PhysPort [60].

\section{B. Sample}

The data for this study were collected at a large southern land-grant university serving approximately 25000 students. Overall university undergraduate demographics were 79\% White, 5\% African American, 6\% Hispanic, with other groups each $3 \%$ or less for the period studied [61].

The sample consists of 4509 complete pretest records (3482 men and 1027 women) and 4716 complete posttest records (3628 men and 1088 women) from students enrolled in an introductory calculus-based mechanics course. The class served primarily engineering and physical sciences majors and was presented by the same lead instructor using a consistent pedagogy throughout the study. This instructor implemented interactive engagement activities in the lecture and multiple researchbased instructional methods were used in the required laboratory sections.

\section{Partial correlation}

To allow the investigation of correct and incorrect answers together in the same network, the correlation matrix used by MMA was replaced by the partial correlation matrix. Partial correlation measures the association between two variables while eliminating the effect of one or more other variables. The correlation, $r_{X Y}$, between variable $X$ and variable $Y$ is defined by

$$
r_{X Y}=\frac{E\left[\left(X-\mu_{X}\right)\left(Y-\mu_{Y}\right)\right]}{\sigma_{X} \sigma_{Y}},
$$

where $\mu_{i}$ is the mean of variable $i, \sigma_{i}$ is the standard deviation, and $E[X]$ is the expectation value.

Two variables may be correlated because they are both related to a third variable; partial correlation controls for the relation with a third variable. The partial correlation of variable $X$ and variable $Y$ controlling for variable $Z, r_{X Y \mid Z}$, is defined by

$$
r_{X Y \mid Z}=\frac{r_{X Y}-r_{X Z} r_{Y Z}}{\sqrt{1-r_{X Z}^{2}} \sqrt{1-r_{Y Z}^{2}}},
$$

Conceptually, partial correlation can best be understood in terms of linear regression. If $X, Y$, and $Z$ are continuous random variables and $\epsilon_{X Z}$ are the residuals when $Z$ is regressed on $X$ (controlling $X$ for $Z$ ) and $\epsilon_{Y Z}$ are the residuals when $Z$ is regressed on $Y$, then the partial correlation $r_{X Y \mid Z}$ is the correlation of the residuals, corr $\left(\epsilon_{X Z}, \epsilon_{Y Z}\right)$. The residuals are the part of the variance of $X$ or $Y$ not explained by the variation of $Z$.

The package "ppcor" [62], part of the R software system [9], was used to perform the partial correlation analysis.

\section{Extending modified module analysis}

Modified module analysis uses community detection algorithms on the network defined by the correlation matrix of all incorrect responses with a threshold applied; Study 1 used $r>0.2$, as will this study. Correlation matrix entries below this threshold are set to zero. The nonzero entries represent edges in the network; the edge weight is the strength of the correlation. Study 1 applied a number of additional filters to eliminate insignificant edges and rarely selected nodes; these were repeated. Nodes selected by fewer than 30 students were removed. Edges connecting different responses to the same item (and thus strongly negatively correlated) were removed; the $r>0.2$ threshold naturally removed these edges. Study 1 also removed edges that were not statistically significant after a Bonferroni correction was applied. At the sample size of this study and Study 1 , this removed no additional edges because all edges with $r>0.2$ were significant. A Bonferroni correction divides the significance threshold, for example $p<0.05$, by the number of statistical tests performed to correct for the inflation of type I error. 
To allow MMA to productively identify communities when the correct response nodes are included in the network, the correlation matrix was replaced with the partial correlation matrix controlling for total FCI score. This modified algorithm is called modified module analysis-partial (MMA-P). Study 2 presented both the correlation matrix and the partial correlation matrix of the correct answers to FCI for this sample. The two matrices were very different with the correlation matrix forming a sparsely connected network and the partial correlation matrix separating into small subnetworks.

The partial correlation matrix has some theoretical advantages over the correlation matrix if the goal is to identify consistently applied reasoning. Two items in an instrument can be correlated either because only the highest performing students get them correct or because only the lowest performing students get them wrong; they are correlated due to overall test score. To remove these correlations, the partial correlation matrix controls for overall test score. This explains why in Study 2 the correlation matrix was generally connected with positive correlations, but the partial correlations contained isolated communities; the correlations between items in the FCI are generally related to the total FCI score.

The MMA algorithm was then applied to the partial correlation matrix. The fast-greedy community detection algorithm (CDA) was applied to the network defined by the partial correlation matrix; this identified subsets of the network that were more connected to each other than they were to nodes outside the community. The community structure is sensitive both to random noise in the data and to the stochastic nature of CDAs. To determine the structure not related to these factors, the dataset was bootstrapped with 1000 replications; the CDA was run on each replication. The R package "boot" was used for bootstrapping [63]. As in Study 1, the dataset was down sampled to provide a sample balanced between men and women. The number of times each pair of responses was found in the same community was recorded for each of the 1000 samples forming a "community matrix." The community fraction, $C$, was defined as the fraction of the bootstrap replications in which node $i$ and $j$ were in the same community. Only nodes found in $C>80 \%$ of the communities were analyzed.

\section{RESULTS}

\section{A. Extended modified module analysis}

Modified module analysis-Partial was applied to understand the structure of the FCI. The communities detected with a partial correlation threshold of $r>0.2$ identified in $80 \%$ of the bootstrap replications $(C>80 \%)$ are shown in Table I. The communities are represented graphically in Fig. 1. Nodes representing correct responses are identified with an asterisk (*). Node placement is for visual effect only; however, the placement algorithm places more strongly correlated nodes near each other. The communities naturally divide into three classes as shown in Table I: communities formed of only correct answers, communities formed of only incorrect answers, and communities that mix correct and incorrect answers. Each type of community is examined below. Some communities identified by MMA-P were also identified in Study 1 and are marked $\otimes$, some were only identified in this study and are marked $\times$, some were only identified in Study 1 and are marked $\bigcirc$; some communities identified in Study 1 merged with correct answers to formed mixed correctincorrect communities and are marked $\odot$.

Figure 1 is a refinement of the network visualization used in Study 1 and in Wells et al. [15]. In these previous studies, a line was drawn between the two nodes if the nodes were identified in the same community $80 \%$ of the time $(C>80 \%)$; however, two nodes can be found in the same community because they are always connected to a third node. For example, in Fig. 1(c) responses $\left\{21 \mathrm{E}^{*}, 22 \mathrm{~B}^{*}, 26 \mathrm{E}^{*}\right\}$ were detected in the same community in at least $80 \%$ of the replications; however, $21 \mathrm{E}^{*}$ and $26 \mathrm{E}^{*}$ are weakly correlated. Both are strongly correlated with $22 \mathrm{~B}^{*}$. To provide a better indication of the degree of connection of two nodes in the individual bootstrap replications of the CDA, the average correlation of the two nodes across the 1000 replicates is used as the edge weight in Fig. 1. The thickness of the edge is proportional to the correlation.

The degree of connection of a community is characterized by the intracommunity density $\gamma$, the ratio of the number of edges observed to the maximum possible number of edges [64]. For example, a community that contains four nodes can be connected with a maximum of six edges. If only four of those edges are observed, then $\gamma=4 / 6$. For communities that are not fully connected $(\gamma<1), \gamma$ is presented in parentheses in Table I.

\section{B. The structure of the response communities \\ 1. Completely incorrect communities}

The original MMA algorithm could only explore incorrect responses, and therefore, all communities reported in Study 1 were composed of incorrect responses. The incorrect communities identified by MMA and MMA-P were very similar but not identical.

Study 1 showed that there were two classes of incorrect communities. One was related to the practice of blocking items; for other communities, the students seemed to be applying consistent incorrect reasoning, a misconception.

Communities $\{8 \mathrm{~A}, 9 \mathrm{~B}\},\{21 \mathrm{~B}, 23 \mathrm{C}\}$, and $\{21 \mathrm{C}, 22 \mathrm{~A}\}$ are answers within blocked problems where the second answer in the pair would be correct if the first answer was correct. These were fairly consistently detected by both MMA and MMA-P; however, the $\{21 \mathrm{C}, 22 \mathrm{~A}\}$ community merged with some correct responses to form a mixed 
TABLE I. Communities identified in the pretest and post-test at $r>0.2$ and $C>80 \%$. The number in parentheses is the intracommunity density, $\gamma$, for communities where the intracommunity density is not 1 . Communities labeled $\otimes$ were identified in both Study 1 and the current study. Communities labeled $\bigcirc$ were identified in Study 1 but not in the current study. Communities labeled $\times$ were identified in the current study, but not in Study 1. Communities labeled $\odot$ were identified in Study 1 as completely incorrect communities, but combined with correct responses to form mixed correct-incorrect communities in the current study.

\begin{tabular}{|c|c|c|c|c|c|}
\hline \multirow[b]{2}{*}{ Community } & \multicolumn{2}{|c|}{ Pretest } & \multicolumn{2}{|c|}{ Post-test } & \multirow[b]{2}{*}{ Misconception, principle, explanation } \\
\hline & Men & Women & Men & Women & \\
\hline \multicolumn{6}{|c|}{ Completely incorrect communities } \\
\hline $1 \mathrm{~A}, 2 \mathrm{C}$ & $\odot$ & & & & Heavier objects fall faster. \\
\hline $1 \mathrm{D}, 2 \mathrm{D}$ & $\otimes$ & & & & Lighter objects fall faster. \\
\hline $4 \mathrm{~A}, 15 \mathrm{C}, 28 \mathrm{D}$ & $\otimes$ & $\otimes$ & $\odot$ & $\odot$ & Newton's 3rd law misconceptions. \\
\hline $5 \mathrm{D}, 18 \mathrm{D}$ & & & $\otimes$ & $\times$ & Motion implies active forces. \\
\hline $5 \mathrm{D}, 11 \mathrm{C}, 13 \mathrm{C}, 18 \mathrm{D}, 30 \mathrm{E}$ & & & & & Motion implies active forces. \\
\hline $5 \mathrm{E}, 18 \mathrm{E}$ & $\otimes$ & $\otimes$ & $\otimes$ & $\bigcirc$ & Motion implies active forces, centrifugal force. \\
\hline $6 \mathrm{~A}, 7 \mathrm{~A}$ & $\bigcirc$ & $\otimes$ & $\otimes$ & $\otimes$ & Circular impetus. \\
\hline $8 \mathrm{~A}, 9 \mathrm{~B}$ & $\otimes$ & $\otimes$ & $\otimes$ & $\otimes$ & Blocked item. \\
\hline $11 \mathrm{~B}, 29 \mathrm{~A}$ & $\otimes$ & $\bigcirc$ & & & Motion implies active forces. \\
\hline $11 \mathrm{C}, 13 \mathrm{C}$ & & & $x$ & $x$ & Motion implies active forces. \\
\hline $11 \mathrm{C}, 13 \mathrm{C}, 30 \mathrm{E}$ & & & $\bigcirc$ & & Motion implies active forces. \\
\hline $15 \mathrm{D}, 16 \mathrm{D}$ & $x$ & & & & Newton's 3rd law misconceptions. \\
\hline $17 \mathrm{~A}, 25 \mathrm{D}$ & & & $\otimes$ & $\bigcirc$ & Largest force determines motion. \\
\hline $21 \mathrm{~B}, 23 \mathrm{C}$ & & & $\otimes$ & $\otimes$ & Blocked item. \\
\hline $21 \mathrm{C}, 22 \mathrm{~A}$ & $\otimes$ & & $\odot$ & $\odot$ & Blocked item. \\
\hline $23 \mathrm{D}, 24 \mathrm{C}$ & $\otimes$ & $\otimes$ & $\otimes$ & $\otimes$ & Impetus dissipation. \\
\hline \multicolumn{6}{|c|}{ Mixed correct and incorrect communities } \\
\hline $1 \mathrm{~A}, 2 \mathrm{C}, 17 \mathrm{~B} *$ & $x$ & & & & Unknown \\
\hline $4 \mathrm{~A}, 14 \mathrm{D} *, 15 \mathrm{C}, 28 \mathrm{D}$ & & & & $\times(67 \%)$ & Newton's 3rd law and 2D kinematics. \\
\hline $4 \mathrm{~A}, 15 \mathrm{C}, 21 \mathrm{E}^{*}, 22 \mathrm{~B} *, 28 \mathrm{D}$ & & & $x$ & & Newton's 3rd law and 2D kinematics. \\
\hline $8 \mathrm{~B}^{*}, 9 \mathrm{E}^{*}, 15 \mathrm{~A}^{*}, 21 \mathrm{C}, 22 \mathrm{~A}$ & & & & $x$ & $\begin{array}{l}8 \mathrm{~B} \text { and } 21 \mathrm{C} \text { share a similar trajectory; } 8 \mathrm{~B} \text { and } \\
9 \mathrm{E} \text { are blocked. }\end{array}$ \\
\hline $8 \mathrm{~B}, 21 \mathrm{C}^{*}$ & & $x$ & & & $8 \mathrm{~B}$ and $21 \mathrm{C}$ share a similar trajectory. \\
\hline $8 \mathrm{~B} *, 21 \mathrm{C}, 22 \mathrm{~A}$ & & & $x$ & & $8 \mathrm{~B}$ and $21 \mathrm{C}$ share a similar trajectory. \\
\hline $23 \mathrm{~A}, 24 \mathrm{~A}^{*}$ & & $x$ & & & $24 \mathrm{~A}$ is the correct answer if $23 \mathrm{~A}$ were correct. \\
\hline \multicolumn{6}{|c|}{ Completely correct communities } \\
\hline $4 \mathrm{E}^{*}, 28 \mathrm{E}^{*}$ & & & & $\times$ & Newton's 3rd law. \\
\hline $4 \mathrm{E}^{*}, 15 \mathrm{~A}^{*}, 28 \mathrm{E}^{*}$ & & $\times(67 \%)$ & $x$ & & Newton's 3rd law. \\
\hline $5 \mathrm{~B}^{*}, 18 \mathrm{~B}^{*}$ & & & $x$ & $x$ & Centripetal acceleration in a curved trajectory. \\
\hline $6 \mathrm{~B} *, 7 \mathrm{~B} *$ & & & $x$ & $x$ & $\begin{array}{l}\text { Instantaneous velocity is tangent to the } \\
\text { trajectory. }\end{array}$ \\
\hline $11 D^{*}, 13 D^{*}$ & & & & $x$ & $\begin{array}{l}\text { Motion under gravity; a force in the direction of } \\
\text { motion is not necessary. }\end{array}$ \\
\hline $15 \mathrm{~A}^{*}, 28 \mathrm{E}^{*}$ & $x$ & & & & Newton's 3rd law. \\
\hline $17 \mathrm{~B}^{*}, 25 \mathrm{C}^{*}$ & & $x$ & & $x$ & Newton's 1st law; Addition of forces. \\
\hline $17 \mathrm{~B}^{*}, 25 \mathrm{C}^{*}, 26 \mathrm{E}^{*}$ & & & $x$ & & $\begin{array}{l}\text { Newton's 1st and 2nd law; Addition of forces; } \\
\text { (26E) 1D acceleration. }\end{array}$ \\
\hline $21 \mathrm{E}^{*}, 22 \mathrm{~B} *, 26 \mathrm{E}^{*}$ & & & & $x$ & Newton's 2nd law; 1D and 2D kinematics. \\
\hline
\end{tabular}

community post-instruction for both men and women in MMA-P. This occurred because, postinstruction, the community joined with response $8 \mathrm{~B}^{*}$ for men and responses $8 \mathrm{~B}^{*}, 9 \mathrm{E}^{*}$, and $15 \mathrm{~A}$ for women. These new communities are discussed with the mixed correct and incorrect communities.

Three incorrect communities were identified by MMA-P, but not MMA. In community $\{15 \mathrm{D}, 16 \mathrm{D}\}$, both items are incorrect responses to the Newton's 3rd law group identified by Study 2; however, they are also part of an item block. It is, therefore, impossible to determine if the community results from a shared misconception or from the effect of blocking. Item 16 is inconsistently identified with other Newton's 3rd law items in exploratory factor analysis studies $[3,16,18-20,65,66]$. The two other incorrect communities identified by MMA-P but not MMA resulted from splitting the $\{5 \mathrm{D}, 11 \mathrm{C}, 13 \mathrm{C}, 18 \mathrm{D}, 30 \mathrm{E}\}$ community into the communities $\{5 \mathrm{D}, 18 \mathrm{D}\}$ and $\{11 \mathrm{C}, 13 \mathrm{C}\}$. 


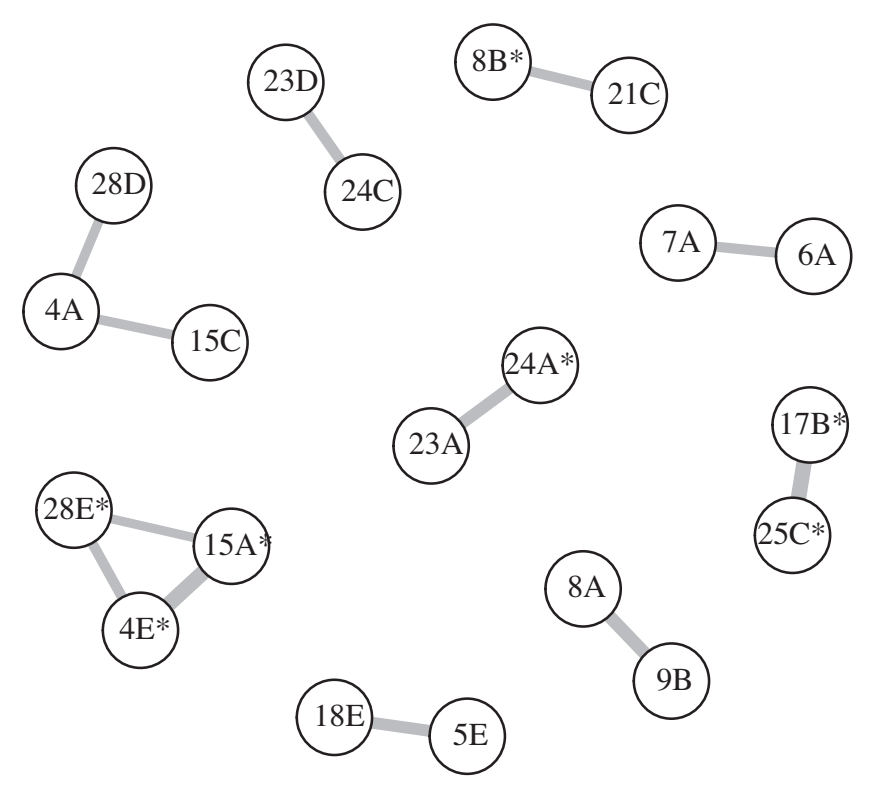

(a) Women - Pretest

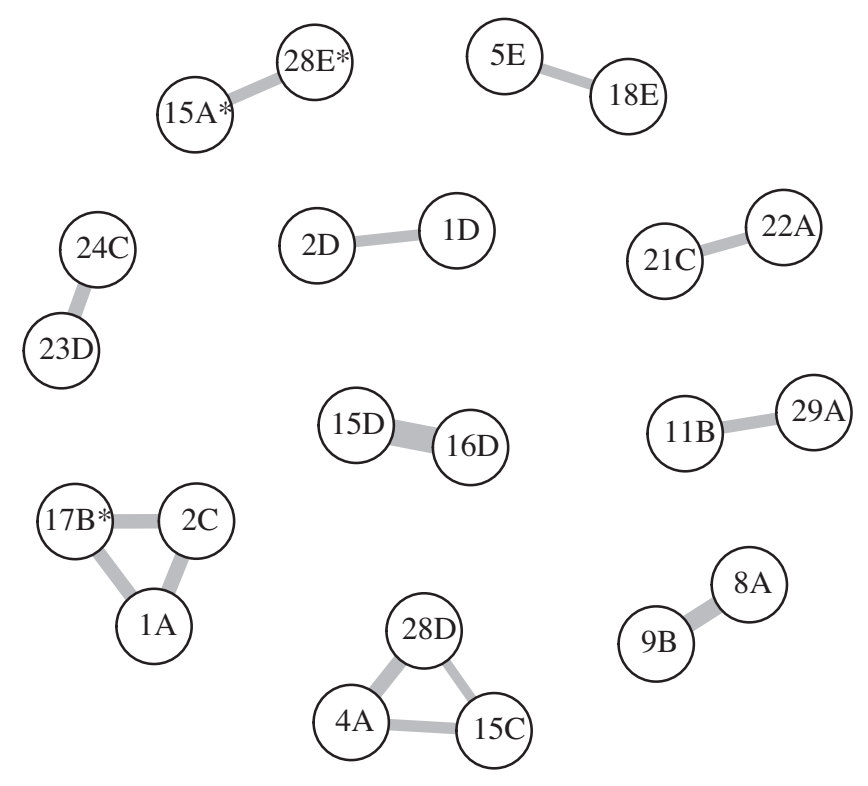

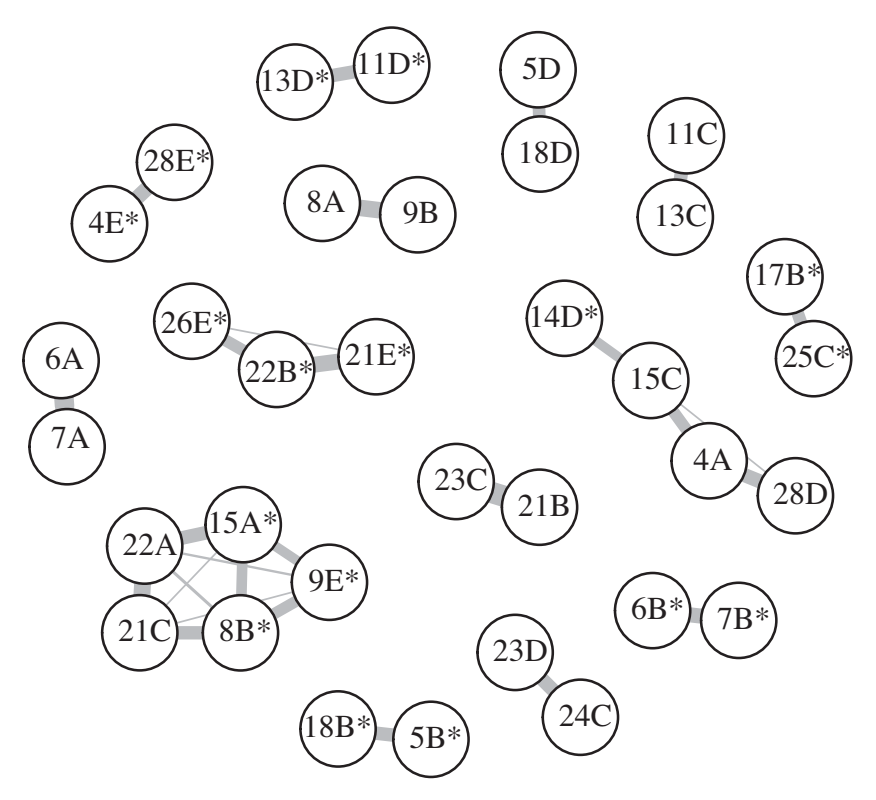

(c) Women - Post-test

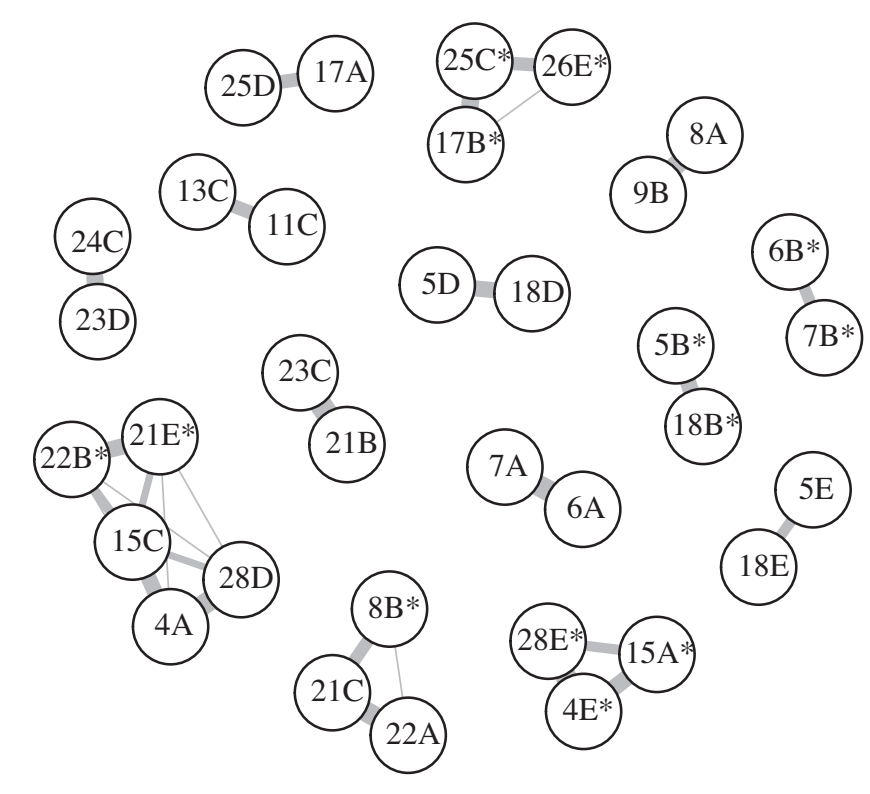

(d) Men - Post-test

FIG. 1. Communities detected in the FCI partial correlation matrix with $r>0.2$. The strength of the correlation is represented by the line thickness.

\section{Completely correct communities}

Study 2 identified four groups of items requiring similar solution structure: $\{4,15,16,28\},\{5,18\},\{6,7\}$, and $\{17,25\}$. Three of the completely correct communities combine items in the first group, $\left\{4 \mathrm{E}^{*}, 15 \mathrm{~A}^{*}, 28 \mathrm{E}^{*}\right\}$, $\left\{4 \mathrm{E}^{*}, 28 \mathrm{E}^{*}\right\}$, and $\left\{15 \mathrm{~A}^{*}, 28 \mathrm{E}^{*}\right\}$; all of which require Newton's 3rd law for their solution. All items in the three-item communities are strongly correlated in Fig. 1.
Correct communities $\left\{5 \mathrm{~B}^{*}, 18 \mathrm{~B}^{*}\right\},\left\{6 \mathrm{~B}^{*}, 7 \mathrm{~B}^{*}\right\}$, and $\left\{17 \mathrm{~B}^{*}, 25 \mathrm{C}^{*}\right\}$ were all identified as requiring similar reasoning for their solution in Study 2. We note the prevalence of the $\mathrm{B}$ response is a result of the extremely unbalanced use of distractors in the FCI [67]. The correct community $\left\{17 \mathrm{~B}^{*}, 25 \mathrm{C}^{*}, 26 \mathrm{E}^{*}\right\}$ extends the community $\left\{17 \mathrm{~B}^{*}, 25 \mathrm{C}^{*}\right\}$ by adding item 26 . Both items 17 and 25 require the addition of forces and Newton's 1st law for their 
solution. Item 26 requires the addition of forces, but the forces are unbalanced, requiring Newton's 2nd law and one-dimensional kinematics (a net force in the direction of motion causes an object to speed up). Because the correct solution structure is substantially different, it seems likely this community is detected because items 25 and 26 are in the same item block. This is supported by Fig. 1(d) where there is a weak correlation between responses $17 \mathrm{~B}^{*}$ and $26 \mathrm{E}^{*}$ in the community $\left\{17 \mathrm{~B}^{*}, 25 \mathrm{C}^{*}, 26 \mathrm{E}^{*}\right\}$.

Both items in the correct community $\left\{11 \mathrm{D}^{*}, 13 \mathrm{D}^{*}\right\}$ ask the students to identify the forces on an object in motion; gravity and a normal force for item 11 and gravity alone for item 13 . Both also explicitly test the motion implies active forces misconception. Item 11 was eliminated from the constrained MIRT analysis in Study 2 because of blocking and, therefore, no item level measure of the discrimination of the item on the use of the normal force is available. With the identification of the correct community, $\left\{11 \mathrm{D}^{*}, 13 \mathrm{D}^{*}\right\}$, it seems likely that both items largely test knowledge of the existence of a downward force of gravity while in motion.

The correct community $\left\{21 \mathrm{E}^{*}, 22 \mathrm{~B}^{*}, 26 \mathrm{E}^{*}\right\}$ contained blocked items 21 and 22 along with item 26. Items 21 and 22 , while blocked, test fairly different physical principles; however, items 22 and 26 both require the principle that if a force is applied in the direction of motion, an object will speed up. There are strong correlations between responses $21 \mathrm{E}^{*}$ and $22 \mathrm{~B}^{*}$ and between responses $22 \mathrm{~B}^{*}$ and $26 \mathrm{E}^{*}$ in Fig. 1(c), but a weak correlation between $21 \mathrm{E}^{*}$ and $26 \mathrm{E}^{*}$. It seems likely blocking caused a relation of items 21 and 22 and the shared principle produced the relation between items 22 and 26.

\section{Mixed correct and incorrect communities}

In addition to examining the grouping of incorrect or correct items, MMA-P can also detect communities combining correct and incorrect items. These communities can provide further insight into the functioning of the FCI and the complexities of student thinking.

Many of the mixed communities seem to form because a pair of questions mixes a correct and an incorrect community. For example, responses $\left\{8 \mathrm{~B}^{*}, 21 \mathrm{C}\right\}$ both represent the same trajectory (a parabolic curve); this trajectory is the correct answer to item 8 and the incorrect answer to item 21 . This similarity serves to explain the mixed communities $\left\{8 \mathrm{~B}^{*}, 21 \mathrm{C}\right\}$ and $\left\{8 \mathrm{~B}^{*}, 21 \mathrm{C}, 22 \mathrm{~A}\right\}$. Items 21 and 22 are blocked items where response $22 \mathrm{~A}$ is the correct response if response $21 \mathrm{C}$ had been the correct response. Responses $8 \mathrm{~B}^{*}$ and 22A are weakly related in Fig. 1(d) making it more likely that the shared trajectory is the cause of the community. If a student is selecting a specific trajectory using both the correct and incorrect reasoning, it may indicate that response $8 \mathrm{~B}^{*}$ is being selected correctly without a solid understanding.

The relation of $8 \mathrm{~B}^{*}$ and $21 \mathrm{C}$ partially explains the community $\left\{8 \mathrm{~B}^{*}, 9 \mathrm{E}^{*}, 15 \mathrm{~A}^{*}, 21 \mathrm{C}, 22 \mathrm{~A}\right\}$; items 8 and 9 are blocked and to answer item 9 correctly requires a correct response to item 8. It is unclear why Newton's 3rd law item 15 is associated with this group. Some items in this group are weakly correlated as shown in Fig. 1(c), but the strong relation of $15 \mathrm{~A}^{*}$ to $8 \mathrm{~B}^{*}, 9 \mathrm{~B} *$, and $22 \mathrm{~A}$ does not have a grounding in either the correct solution structure of the items or a shared misconception. It is also difficult to identify a $p$ prim that might have been activated in each case. Items 21 and 22 were identified as substantially unfair to women in Traxler et al. [13]. The relation of the correct response $15 \mathrm{~A} *$ to $22 \mathrm{~A}$ and correct response $8 \mathrm{~B}^{*}$ to $21 \mathrm{C}$ correcting for overall test score provides evidence that these items are being incorrectly answered for reasons not completely related to Newtonian reasoning ability.

Responses 21E* and 22B* are part of an item block. The identification of these items in a community of incorrect Newton's 3rd law responses, \{4A, 15C, 28D for men, may indicate that men are selecting the items correctly for the wrong reasons which would partially explain the unfairness of items 21 and 22 reported by Traxler et al. [13].

It is possible a similar effect explains the community $\left\{4 \mathrm{~A}, 14 \mathrm{D}^{*}, 15 \mathrm{C}, 28 \mathrm{D}\right\}$. If students are selecting correct response $14 \mathrm{D}^{*}$ for incorrect reasons, it could be more correlated with the incorrect Newton's 3rd law items than would be predicted by the overall FCI score. This community was only identified for women postinstruction. Item 14 was one of the items identified as substantially unfair to women in Traxler et al. [13]; if it is also being answered correctly by women for incorrect reasons, it may be more unfair than previously reported.

Responses $23 \mathrm{~A}$ and $24 \mathrm{~A}^{*}$ are also part of an item block. Item $23 \mathrm{~A}$ tests the misconception of impetus dissipation where after a force is removed the object returns to its original trajectory before the force was applied. Item 24A* (constant speed) is the correct answer for the correct reasoning for item 23, but it is also the correct answer if the misconception of impetus dissipation actually applied. This suggests that some students are getting item 24 correct for the wrong reasons, and that the item block may need to be restructured.

The community $\left\{1 \mathrm{~A}, 2 \mathrm{C}, 17 \mathrm{~B}^{*}\right\}$ all involve items with an object moving under the force of gravity. There is little additional relation between the items. It is difficult to make a theoretical case for this community either because of a shared misconception or the correct answer structure. It may be that these items, identified as a community only for men on the pretest, use less consistent reasoning better modeled using the knowledge-in-pieces framework as a pprim. Response 1A might be answered by applying the "heavier is faster" principle, response $2 \mathrm{C}$ the "heavier travels farther" principle, and response 17B* the "constant implies equal" principles; all examples of a fragment of reasoning that "like implies like." 


\section{DISCUSSION}

\section{A. Research questions}

This study sought to answer three research questions; they will be addressed in the order proposed.

RQ1: How can modified module analysis be extended to include correct responses?

Modified module analysis was extended to include correct answers by replacing the correlation matrix with the partial correlation matrix correcting for overall FCI test score. The modified algorithm was called modified module analysis-partial (MMA-P). This modification allowed the identification of a number of relatively small communities of correct and incorrect answers producing a network quantitatively similar to the MMA algorithm, but including correct answers.

RQ2: Are the communities detected by the extended algorithm productive in furthering the understanding of the FCI? How are the communities identified by the extended algorithm related to structures identified in previous studies?

Study 1 could only report completely incorrect communities. Table I provides a comparison between the two studies. Of the 38 communities identified either on the pretest or post-test for men or women, 24 were identified in both studies, 11 were identified only in Study 1, and 3 only in the present study. Four of the communities identified in only Study 1 merged with correct answers to form mixed communities in this study. As such, MMA and MMA-P produce fairly similar incorrect answer communities. The remaining incorrect communities identified in Study 1, but not in the present study, may have resulted from correlations with overall test score where only very weak or very strong students selected the items.

Until this study, module analysis was not productive for finding communities of correct answers in the FCI. Study 2 presented both the FCI correlation matrix and the partial correlation matrix for the correct answers to the FCI for the dataset used in this study. The correlation matrix was sparsely and randomly interconnected and showed little community structure. Clear community structure was evident in the partial correlation matrix. As such, it was not surprising that modifying MMA to use the partial correlation matrix allowed the extraction of compact correct answer communities.

Study 2 did not use network analysis to form communities of correct answers, but it did present a taxonomy of isomorphic and blocked problems that can be compared with the community structure. Two items are isomorphic if they required the same reasoning process for their solution. Many of the components of the correct communities in Table I were identified in Study 2 as having similar solution structure $\left\{4 \mathrm{E}^{*}, 15 \mathrm{~A}^{*}, 28 \mathrm{E}^{*}\right\}$, $\left\{5 \mathrm{~B}^{*}, 18 \mathrm{~B}^{*}\right\},\left\{6 \mathrm{~B}^{*}, 7 \mathrm{~B}^{*}\right\}$, and $\left\{17 \mathrm{~B}^{*}, 25 \mathrm{C}^{*}\right\}$. The students do not consistently integrate the Newton's 3rd law items, $\left\{4 \mathrm{E}^{*}, 15 \mathrm{~A}^{*}, 28 \mathrm{E}^{*}\right\}$, with different combinations observed for men and women either on the pretest or post-test. The items in community $\left\{11 \mathrm{D}^{*}, 13 \mathrm{D}^{*}\right\}$ differ only by the addition of the normal force; both items specifically test the motion implies active forces misconception by providing a distractor which indicates there is a force in the direction of motion. The incorrect community, $\{11 \mathrm{C}, 13 \mathrm{C}\}$, of students who apply this misconception was also identified. The addition of the normal force does not seem an important discriminating factor in whether the items are answered correctly.

The attachment of $26 \mathrm{E}^{*}$ to the $\left\{17 \mathrm{~B}^{*}, 25 \mathrm{C}^{*}\right\}$ community of isomorphic items for men likely resulted from the blocking of items 25 and 26; the solution structure of item 26 does not suggest it should be directly associated with the other two items. The correlations in Fig. 1 support this interpretation. Item $26 \mathrm{E}^{*}$ is also associated with the blocked items $21 \mathrm{~B}^{*}$, and $22 \mathrm{~B}^{*}$ for women; however, the association is much stronger with item $22 \mathrm{~B}^{*}$; both item 22 and 26 require application of the principle that forces in the direction of motion cause objects to speed up.

As such, the correct community structure is largely what was suggested by Study 2 with some additional sets of items that are being answered consistently which were not modeled as having similar structure in Study 2. Some of the connections identified continue to support the negative impacts of blocking items on the interpretability of the results of the instrument.

No prior studies have examined community structure combining incorrect and correct responses in the same community. The mixed communities identified in this study seem to originate largely from combinations of items that may not be working as intended in the instrument; items that are being answered correctly for the wrong reason or items that are possibly being misinterpreted contributing to instrumental unfairness.

RQ3: How do the incorrect communities change between the pretest and the post-test? RQ4: How do the communities detected differ between men and women? The differences from pretest to post-test differ for men and women, and therefore, these two questions will be taken together. Table I shows the communities identified by MMA-P. The completely correct, completely incorrect, and mixed correct-incorrect communities are discussed independently.

For completely incorrect communities, incorrect communities identified in Study 1 which merged with correct answers to form mixed communities in this study (labeled $\odot$ in Table I) will be included in the incorrect communities; the incorrect items in the community were still detected in the same community. For men, 9 communities were detected on the pretest and 10 on the post-test; only 5 were consistent from pretest to post-test. For women, 5 communities were identified on the pretest and 8 on the post-test; 4 were consistent between the pretest and 
post-test. As such, women have more consistent incorrect reasoning patterns between pretest to post-test, but men have more communities of incorrect reasoning both preinstruction and postinstruction.

Comparing men and women, only 4 of the 10 pretest communities were consistent for men and women, while 8 of the 10 post-test communities were consistent between men and women. As such, there is little consistency in incorrect reasoning between men and women on the pretest, but substantial consistency on the post-test. Men and women also had a similar number of items transition from completely incorrect in Study 1 to mixed correctincorrect in this study.

Six incorrect communities identified by MMA in Study 1 were not identified by MMA-P in this study. These missing communities were often related to new communities identified by MMA-P but not MMA. The community $\{11 \mathrm{C}, 13 \mathrm{C}, 30 \mathrm{E}\}$ identified by MMA became the community $\{11 \mathrm{C}, 13 \mathrm{C}\}$ using MMA-P for men on the post-test. Response $11 \mathrm{C}$ and $13 \mathrm{C}$ test the force in the direction of motion misconception while response $30 \mathrm{E}$ asks about the "force of a hit;" as such, the dissolution of the community is understandable. The community \{5D, 11C, 13C, 18D, 30E $\}$ identified by MMA became the communities $\{5 \mathrm{D}, 18 \mathrm{D}\}$ and $\{11 \mathrm{C}, 13 \mathrm{C}\}$ in MMA$\mathrm{P}$; both can be theoretically justified using the framework of Study 2 and both test similar misconceptions in the framework of Hestenes and Jackson [14]. It may be the identification of the $\{5 \mathrm{D}, 11 \mathrm{C}, 13 \mathrm{C}, 18 \mathrm{D}, 30 \mathrm{E}\}$ was a result of correlations generated by overall test score.

Four of the communities identified by MMA but not MMA-P were more difficult to explain. Communities $\{6 \mathrm{~A}, 7 \mathrm{~A}\},\{5 \mathrm{E}, 18 \mathrm{E}\}$, and $\{17 \mathrm{~A}, 25 \mathrm{D}\}$ contain incorrect responses to items identified in Study 2 as having the same correct solution structure. It is unclear why the incorrect reasoning for these items is not applied consistently. The correct answer communities for these items were consistently identified postinstruction. It may be that students are transitioning to correct reasoning and that the misconception is no longer consistently applied. Pretest community $\{11 \mathrm{~B}, 29 \mathrm{~A}\}$ also was no longer identified for women; students giving responses in this community fail to account for the normal force. It is unclear why women do not do so consistently. Item 29 was identified by Traxler et al. [13] as having poor psychometric properties, so it may be that the item is simply not functioning well.

Fewer mixed communities than incorrect communities were detected either preinstruction or post-instruction for men or women. There were no consistent mixed communities either from pretest to post-test or for men and women.

Only three correct communities were detected on the pretest for either men or women; only one was also identified postinstruction. Most correct communities were detected postinstruction; four for men and six for women; only two of these communities were consistent between men and women. One of the inconsistent correct communities $\left\{17 \mathrm{~B}^{*}, 25 \mathrm{C}^{*}, 26 \mathrm{E}^{*}\right\}$ likely resulted from blocking items 25 and 26; if response $26 \mathrm{E}^{*}$ were removed the community $\left\{17 \mathrm{~B}^{*}, 25 \mathrm{C}^{*}\right\}$ would also be consistent between men and women. Three of the other inconsistent communities result from the inconsistent application of Newton's 3rd law. Responses 4E* $15 \mathrm{~A}^{*}$, and $28 \mathrm{E}^{*}$ were identified in the same community and were highly correlated [Fig. 1(d)] for men on the post-test. Only responses $4 \mathrm{E}^{*}$ and $28 \mathrm{E}^{*}$ were in the same community for women; response $15 \mathrm{~A}^{*}$ was identified as part of a mixed community for women. If the minor differences in the $\left\{4 \mathrm{E}^{*}, 15 \mathrm{~A}^{*}, 28 \mathrm{E}^{*}\right\}$ and $\left\{17 \mathrm{~B}^{*}, 25 \mathrm{C}^{*}\right\}$ communities are ignored, then all correct communities identified for men postinstruction were also identified for women; two additional correct communities were identified for women. The items in one of the additional communities, $\left\{11 \mathrm{D}^{*}, 13 \mathrm{D}^{*}\right\}$, differ by the inclusion of a normal force; this difference affects men but not women. The last inconsistent community $\left\{21 \mathrm{E}^{*}, 22 \mathrm{~B}^{*}\right.$, and $\left.26 \mathrm{E}^{*}\right\}$ involves the blocked items 21 and 22 , but also item 26 which requires somewhat different physical reasoning. It is unclear why these items are identified in the same community; however, items 21 and 22 were two items identified as highly unfair to women in Traxler et al. [13].

\section{B. Other observations}

The MMA-P algorithm identified much of the incorrect structure identified by MMA while also productively identifying correct structure. The structures identified were consistent with the theoretical framework provided by Study 2 and with the taxonomy of misconceptions of Hestenes and Jackson [14]. The mixed correct-incorrect communities allowed the identification of combinations of responses where symmetric correct and incorrect reasoning was applied suggesting the correct response was being selected for the incorrect reason. These communities also provide some hint as to items within the FCI which were not functioning correctly where correct answers were related to a community of consistently applied misconceptions. In general, the MMA-P extension of MMA provided a richer picture of the instrument.

\section{FUTURE}

The MMA-P algorithm will be applied to other popular conceptual instruments such as the FMCE, the Conceptual Survey of Electricity and Magnetism [68], and the Brief Electricity and Magnetism Assessment [69] to further understand their structure. The algorithm will also be applied to multiple samples taken from students at different institutions to understand how the incorrect reasoning structures identified change with institutional setting. 


\section{CONCLUSION}

This work extended the modified module analysis algorithm of Wells et al. [5] to allow the analysis of correct and incorrect responses simultaneously creating modified module analysis - partial (MMA-P). MMA-P applied the same methodology as MMA, but to the partial correlation matrix rather than the correlation matrix. This change allowed MMA-P to identify incorrect communities as did MMA, but also fully correct communities and communities that mixed correct and incorrect responses. The incorrect communities identified were generally consistent with those identified by MMA. The correct communities generally followed those identified by Stewart et al. [16]; therefore, MMA-P productively identified both correct solution structure and incorrect structure at the same time. MMA-P also identified communities which mixed correct and incorrect answers. These communities were productive in understanding answering patterns where the correct answer was possibly selected for the incorrect reason and in understanding some of the gender unfairness identified by Traxler et al. The post-test reasoning of men and women was generally consistent.

\section{ACKNOWLEDGMENTS}

This work was supported in part by the National Science Foundation as part of the evaluation of improved learning for the Physics Teacher Education Coalition, PHY0108787.
[1] D. Hestenes, M. Wells, and G. Swackhamer, Force Concept Inventory, Phys. Teach. 30, 141 (1992).

[2] R. K. Thornton and D. R. Sokoloff, Assessing student learning of Newton's laws: The Force and Motion Conceptual Evaluation and the evaluation of active learning laboratory and lecture curricula, Am. J. Phys. 66, 338 (1998).

[3] D. Huffman and P. Heller, What does the Force Concept Inventory actually measure?, Phys. Teach. 33, 138 (1995).

[4] E. Brewe, J. Bruun, and I. G. Bearden, Using module analysis for multiple choice responses: A new method applied to Force Concept Inventory data, Phys. Rev. Phys. Educ. Res. 12, 020131 (2016).

[5] J. Wells, R. Henderson, J. Stewart, G. Stewart, J. Yang, and A. Traxler, Exploring the structure of misconceptions in the Force Concept Inventory with modified module analysis, Phys. Rev. Phys. Educ. Res. 15, 020122 (2019).

[6] S. Sircar and N. Parekh, Functional characterization of drought-responsive modules and genes in Oryza sativa: A network-based approach, Front. Gen. 6, 1 (2015).

[7] J. A. Smith and R. Faris, Movement without mobility: Adolescent status hierarchies and the contextual limits of cumulative advantage, Soc. Networks 40, 139 (2015).

[8] G. Csardi and T. Nepusz, The igraph software package for complex network research, Inter Journal, Complex Systems 1695, 1 (2006).

[9] R Core Team, R: A Language and Environment for Statistical Computing (R Foundation for Statistical Computing, Vienna, Austria, 2017).

[10] S. Salehi, E. Burkholder, G. P. Lepage, S. Pollock, and C. Wieman, Demographic gaps or preparation gaps?: The large impact of incoming preparation on performance of students in introductory physics, Phys. Rev. Phys. Educ. Res. 15, 020114 (2019).

[11] R. Henderson and J. Stewart, Racial and ethnic bias in the Force Concept Inventory, in Proceedings of the Physics Education Research Conference 2017, Cincinnati, OH, edited by L. Ding, A. Traxler, and Y. Cao (AIP, New York, 2017), pp. 172-175.

[12] A. Madsen, S. B. McKagan, and E. Sayre, Gender gap on concept inventories in physics: What is consistent, what is inconsistent, and what factors influence the gap?, Phys. Rev. Phys. Educ. Res. 9, 020121 (2013).

[13] A. Traxler, R. Henderson, J. Stewart, G. Stewart, A. Papak, and R. Lindell, Gender fairness within the Force Concept Inventory, Phys. Rev. Phys. Educ. Res. 14, 010103 (2018).

[14] Table II for the Force Concept Inventory (revised from 081695r), http://modeling.asu.edu/R\&E/FCI-RevisedTable-II_ 2010.pdf. Accessed 3/17/2019.

[15] J. Wells, R. Henderson, A. Traxler, P. Miller, and J. Stewart, Exploring the structure of misconceptions in the Force and Motion Conceptual Evaluation with modified module analysis, Phys. Rev. Phys. Educ. Res. 16, 010121 (2020).

[16] J. Stewart, C. Zabriskie, S. DeVore, and G. Stewart, Multidimensional item response theory and the Force Concept Inventory, Phys. Rev. Phys. Educ. Res. 14, 010137 (2018).

[17] J. Yang, C. Zabriskie, and J. Stewart, Multidimensional item response theory and the Force and Motion Conceptual Evaluation, Phys. Rev. Phys. Educ. Res. 15, 020141 (2019).

[18] T. F. Scott, D. Schumayer, and A. R. Gray, Exploratory factor analysis of a Force Concept Inventory data set, Phys. Rev. Phys. Educ. Res. 8, 020105 (2012).

[19] T. F. Scott and D. Schumayer, Students' proficiency scores within multitrait item response theory, Phys. Rev. Phys. Educ. Res. 11, 020134 (2015).

[20] M. R. Semak, R. D. Dietz, R. H. Pearson, and C. W. Willis, Examining evolving performance on the Force Concept Inventory using factor analysis, Phys. Rev. Phys. Educ. Res. 13, 010103 (2017).

[21] C. Nord, S. Roey, S. Perkins, M. Lyons, N. Lemanski, J. Schuknecht, and J. Brown, American High School Graduates: Results of the 2009 NAEP High School Transcript 
Study, U.S. Department of Education (National Center for Education Statistics, Washington, DC, 2011).

[22] B. C. Cunningham, K. M. Hoyer, and D. Sparks, Gender Differences in Science, Technology, Engineering, and Mathematics (STEM) Interest, Credits Earned, and NAEP Performance in the 12th Grade (National Center for Education Statistics, Washington, DC, 2015).

[23] B. C. Cunningham, K. M. Hoyer, and D. Sparks, The Condition of STEM 2016 (ACT Inc., Iowa City, IA, 2016).

[24] Y. Maeda and S. Y. Yoon, A meta-analysis on gender differences in mental rotation ability measured by the Purdue Spatial Visualization Tests: Visualization of Rotations (PSVT: R), Educ. Psychol. Rev. 25, 69 (2013).

[25] D. F. Halpern, Sex Differences in Cognitive Abilities, 4th ed. (Psychology Press, Francis \& Tayler Group, New York, NY, 2012).

[26] J. S. Hyde and M. C. Linn, Gender differences in verbal ability: A meta-analysis, Psychol. Bull. 104, 53 (1988).

[27] J. S. Hyde, E. Fennema, and S. J. Lamon, Gender differences in mathematics performance: A meta-analysis, Psychol. Bull. 107, 139 (1990).

[28] N. M. Else-Quest, J. S. Hyde, and M. C. Linn, Crossnational patterns of gender differences in mathematics: A meta-analysis, Psychol. Bull. 136, 103 (2010).

[29] X. Ma, A meta-analysis of the relationship between anxiety toward mathematics and achievement in mathematics, J. Res. Math. Educ. 30, 520 (1999).

[30] J. V. Mallow and S. L. Greenburg, Science anxiety: Causes and remedies, J. Coll. Sci. Teach. 11, 356 (1982).

[31] M. K. Udo, G. P. Ramsey, and J. V. Mallow, Science anxiety and gender in students taking general education science courses, J. Sci. Educ. Technol. 13, 435 (2004).

[32] J. Mallow, H. Kastrup, F. B. Bryant, N. Hislop, R. Shefner, and M. Udo, Science anxiety, science attitudes, and gender: Interviews from a binational study, J. Sci. Educ. Technol. 19, 356 (2010).

[33] J. R. Shapiro and A. M. Williams, The role of stereotype threats in undermining girls' and women's performance and interest in STEM fields, Sex Roles 66, 175 (2012).

[34] R. D. Dietz, R. H. Pearson, M. R. Semak, and C. W. Willis, Gender bias in the Force Concept Inventory?, AIP Conf. Proc. 1413, 171 (2012).

[35] S. Osborn Popp, D. Meltzer, and M. C. MegowanRomanowicz, Is the Force Concept Inventory biased? Investigating differential item functioning on a test of conceptual learning in physics, in 2011 American Educational Research Association Conference (American Education Research Association, Washington, DC, 2011).

[36] L. McCullough and D. E. Meltzer, Differences in male/ female response patterns on alternative-format versions of the Force Concept Inventory, in Proceedings of the 2001 Physics Education Research Conference, Rochester, NY, edited by K. Cummings, S. Franklin, and J. Marx (AIP, New York, 2001), pp. 103-106.

[37] B. E. Clauser and K. M. Mazor, Using statistical procedures to identify differentially functioning test items, Educ. Meas.-Issues Pra. 17, 31 (1998).

[38] L. Viennot, Spontaneous reasoning in elementary dynamics, Eur. J. Sci. Educ. 1, 205 (1979).
[39] D. E. Trowbridge and L. C. McDermott, Investigation of student understanding of the concept of acceleration in one dimension, Am. J. Phys. 49, 242 (1981).

[40] A. Caramazza, M. McCloskey, and B. Green, Naive beliefs in "sophisticated" subjects: Misconceptions about trajectories of objects, Cognition 9, 117 (1981).

[41] P. C. Peters, Even honors students have conceptual difficulties with physics, Am. J. Phys. 50, 501 (1982).

[42] M. McCloskey, Intuitive physics, Sci. Am. 248, 122 (1983).

[43] R. F. Gunstone, Student understanding in mechanics: A large population survey, Am. J. Phys. 55, 691 (1987).

[44] C. W. Camp and J. J. Clement, Preconceptions in Mechanics: Lessons Dealing with students' Conceptual Difficulties (Kendall/Hunt, Dubuque, IA, 1994).

[45] L. C. McDermott, Students' conceptions and problem solving in mechanics, in Connecting Research in Physics Education with Teacher Education, edited by A. Tiberghien, E. L. Jossem, and J. Barojas (International Commission on Physics Education, Paris, France, 1997), pp. 42-47.

[46] R. Rosenblatt and A. F. Heckler, Systematic study of student understanding of the relationships between the directions of force, velocity, and acceleration in one dimension, Phys. Rev. Phys. Educ. Res. 7, 020112 (2011).

[47] N. Erceg and I. Aviani, Students' understanding of velocity-time graphs and the sources of conceptual difficulties, Croat. J. Educ. 16, 43 (2014).

[48] B. Waldrip, Impact of a representational approach on students' reasoning and conceptual understanding in learning mechanics, Int. J. Sci. Math. Educ. 12, 741 (2014).

[49] A. A. diSessa, Toward an epistemology of physics, Cognit. Instr. 10, 105 (1993).

[50] A. A. diSessa and B. L. Sherin, What changes in conceptual change?, Int. J. Sci. Educ. 20, 1155 (1998).

[51] M. T. H. Chi and J. D. Slotta, The ontological coherence of intuitive physics, Cognit. Instr. 10, 249 (1993).

[52] M. T. H. Chi, J. D Slotta, and N. De Leeuw, From things to processes: A theory of conceptual change for learning science concepts, Learn. Instr. 4, 27 (1994).

[53] J. D. Slotta, M. T. H. Chi, and E. Joram, Assessing students' misclassifications of physics concepts: An ontological basis for conceptual change, Cognit. Instr. 13, 373 (1995).

[54] D. Hammer, Misconceptions or p-prims: How may alternative perspectives of cognitive structure influence instructional perceptions and intentions, J. Learn. Sci. 5, 97 (1996).

[55] D. Hammer, More than misconceptions: Multiple perspectives on student knowledge and reasoning, and an appropriate role for education research, Am. J. Phys. 64, 1316 (1996).

[56] D. Hammer, Student resources for learning introductory physics, Am. J. Phys. 68, S52 (2000).

[57] J. Minstrell, Facets of students' knowledge and relevant instruction, in Research in Physics Learning: Theoretical Issues and Empirical Studies, edited by R. Duit, F. Goldberg, and H. Niedderer (IPN, Kiel, Germany, 1992), pp. $110-128$. 
[58] R. E. Scherr, Modeling student thinking: An example from special relativity, Am. J. Phys. 75, 272 (2007).

[59] I. Halloun, R. R. Hake, E. P. Mosca, and D. Hestenes, Force Concept Inventory (revised 1995), (1995), http:// modeling.asu.edu/R\&E/Research.html. Accessed 7/19/ 2019.

[60] Physport, https://www.physport.org. Accessed 8/8/2017.

[61] US News \& World Report: Education, https://premium .usnews.com/best-colleges. Accessed 4/30/2017.

[62] S. Kim, ppcor: An R package for a fast calculation to semipartial correlation coefficients, Commun. Stat. Appl. M. 22, 665 (2015).

[63] A. Canty and B. D. Ripley, boot: Bootstrap R (S-Plus) Functions (2017), R package version 1.3-20.

[64] K. A. Zweig, Network Analysis Literacy: A Practical Approach to the Analysis of Networks (Springer-Verlag, Wien, Austria, 2016).
[65] D. Hestenes and I. Halloun, Interpreting the Force Concept Inventory: A response to March 1995 critique by Huffman and Heller, Phys. Teach. 33, 502 (1995).

[66] P. Heller and D. Huffman, Interpreting the Force Concept Inventory: A reply to Hestenes and Halloun, Phys. Teach. 33, 503 (1995).

[67] S. DeVore, J. Stewart, and G. Stewart, Examining the effects of testwiseness in conceptual physics evaluations, Phys. Rev. Phys. Educ. Res. 12, 020138 (2016).

[68] D. P. Maloney, T. L. O'Kuma, C. Hieggelke, and A. Van Huevelen, Surveying students' conceptual knowledge of electricity and magnetism, Am. J. Phys. 69, S12 (2001).

[69] L. Ding, R. Chabay, B. Sherwood, and R. Beichner, Evaluating an electricity and magnetism assessment tool: Brief Electricity and Magnetism Assessment, Phys. Rev. ST Phys. Educ. Res. 2, 010105 (2006). 